\title{
LA CONCESIÓN DE LOS MEGAPROYECTOS EN EL PERÚ
}

\section{THE CONCESSION OF MEGAPROJECTS IN PERU}

\author{
Arturo Velásquez Jara*
}

RECEPCIÓN: MAYO DE 2017

\section{RESUMEN}

En el presente artículo, se revisa someramente la experiencia en la concesión de proyectos de infraestructura en el país desde la década de los noventa hasta los últimos años, tiempo en que se han presentado los denominados megaproyectos, lo que ha dado pie a la discusión de si se están logrando las esperadas ventajas de esta modalidad de desarrollo. Del estudio se concluyó que, en una experiencia de veinte años de concesiones en el Perú, lamentablemente, no se han logrado las esperadas ventajas de ahorrar esfuerzos financieros al Estado y tiempo en la mayor parte de los proyectos concesionados. Por ello, se sugiere que, en ciertos casos, sería mejor aplicar el anterior sistema de licitación con financiamiento, el cual existía en la antigua normativa de contrataciones de obras.

La falta de adecuados estudios de preinversión a nivel de factibilidad, sumada al precario estudio de las diversas alternativas de ingeniería conceptual de los proyectos, provoca graves deficiencias en el desarrollo de los mismos. Esta situación ocasiona un incremento innecesario en los costos de inversión previstos en los planes iniciales, además de la falta de previsión oportuna con respecto a la necesidad de expropiaciones, lo que lleva al Estado a iniciar los concursos de selección del concesionario con bases incompletas que se van "madurando" a lo largo de los años.

Finalmente, se recomienda revisar la política y normatividad sobre el desarrollo de proyectos mediante concesiones, y se propone, como requisitos para decidir la concesión de un megaproyecto: 1) que el proyecto cuente con estudios de factibilidad completos, 2) que en ningún caso se permita la aplicación del cofinanciamiento, 3) que los procesos de concurso de concesión cuenten con bases adecuadamente preparadas, y 4) que se declare desierto el concurso para concesión cuando se llegue al final con postor único.

Palabras clave: concesión de proyectos, megaproyectos, financiamiento, licitación con financiamiento, estudios de factibilidad, ingeniería conceptual, bases de concurso, costos de inversión.
ACEPTACIÓN: JUNIO DE 2017

\begin{abstract}
The experience of granting infrastructure projects in the country, from the 1990s to the last years, of the so-called megaprojects is discussed briefly, discussing whether the expected advantages of this type of project development are being achieved.

It is concluded that in the experience of 20 years of concessions in Peru regrettably, the expected advantages of saving financial efforts to the State and saving time in the development of the projects, in most of the concessioned projects, have not been achieved, suggesting that in some cases it would seem better to apply the previous system of tender with financing, which existed in the old regulations for contracting works.

It is argued that the lack of adequate pre-investment studies at the level of feasibility, causes serious shortcomings in the development of the projects, since the various conceptual engineering alternatives of the projects have not been thoroughly studied, causing an unnecessary increase in the investment costs of the projects, in addition to not timely anticipating the need for expropriations, leading to start the concessionaire selection competitions with incomplete bases that are "maturing" over the years.

Finally, it is recommended to review the policy and regulations on the development of projects through concessions, proposing as requirements to decide on the granting of a megaproject: That the project has complete feasibility studies, that the co-financing application is not allowed in any case, that the concession tender processes have adequately prepared bases, and that the tender for concession is declared void when it reaches the end with a single bidder.
\end{abstract}

Keywords: Granting of projects, megaprojects, financing, bidding with financing, feasibility studies, conceptual engineering, tender bases, investment costs.

\footnotetext{
* Profesor principal en la Universidad Ricardo Palma y de la Universidad Nacional de Ingeniería. Cursó estudios de Ingeniería Civil, con segunda especialización en Proyectos de Inversión, maestría en Gerencia de la Construcción Moderna y doctorado en Medio Ambiente y Desarrollo Sostenible. Email: avelasquezjara@yahoo.es
} 


\section{DESARROLLO DEL TEMA}

\section{Breve historia de las concesiones en el Perú}

Cuando en los años noventa se emitieron las primeras normas para la promoción de la inversión privada en obras de infraestructura (DL N ${ }^{\circ} 758$ ), y se introdujo en el país la modalidad de concesiones, esta se presentó como una forma moderna, integral y rápida de desarrollar los proyectos necesarios de inversión. Por ello, muchos estudiosos y preocupados por el desarrollo económico del país se declararon, con mucho entusiasmo, a favor de esta prometedora y nueva modalidad para desarrollar los grandes proyectos a cargo del Gobierno central, y también para desarrollar los proyectos a nivel regional y a nivel de los Gobiernos locales [1]. Sin embargo, durante algunos ańos -aproximadamente, la primera parte de la década de los noventa- no se desarrollaron, en su mayoría, proyectos bajo la modalidad de concesiones, pero luego, al perfeccionarse la normatividad para la aplicación de esta modalidad, a mediados de los noventa (DL N 839 y otras normas), se empezaron a desarrollar algunas concesiones, dentro de las que se puede mencionar la carretera Arequipa-Matarani-Mollendo (1996) y el Terminal Portuario de Matarani (1999), este último con resultados muy favorables porque, al atraer parte importante de la carga boliviana que usaba anteriormente puertos chilenos, permitió elevar muy apreciablemente el volumen de carga movilizado.

En el caso de las autopistas, después de concesionarse la carretera Arequipa-Matarani-Mollendo, tomó varios años concretar otro contrato de concesión, y recién en el año 2002 se otorgó la concesión de la Red Vial No 5, correspondiente al tramo Ancón-Pativilca de la carretera Panamericana. Posteriormente, se fueron concretando algunos otros proyectos viales, pero a un ritmo sumamente lento frente a la urgencia de mejorar la infraestructura de transportes en el país. Ya en el presente siglo, el Estado ha impulsado el desarrollo de importantes proyectos en la nueva modalidad, otorgando la concesión de los denominados megaproyectos tales como los 5 tramos de la IIRSA Sur (del 2005 al 2007), el Sistema Eléctrico de Transporte Masivo de Lima y Callao (Metro) -la Línea 1 el 2011, y la Línea 2 el 2014- y, este último año, el Gasoducto del Sur y el Aeropuerto de Chinchero.

\section{Las esperadas ventajas al concesionar proyectos}

La modalidad de concesionar proyectos de interés público fue considerada como una conveniente forma de lograr la implementación integral de proyectos - con la concurrencia del sector privado- frente a la alternativa de desarrollar los proyectos públicos en la forma tradicional, en la que las etapas se ejecutan de manera separada: estudios, ejecución y operación. Las ventajas que se esperaba obtener en el caso de los proyectos concesionados eran, principalmente, las siguientes:

- Que se traslada la responsabilidad del financiamiento de las inversiones al concesionario. De este modo, se evita que el Estado tenga que asignar recursos de inversión del presupuesto público, por lo que estos quedan disponibles para aplicarlos en proyectos sociales y otras necesidades.

- Que se logra realizar proyectos en plazos más cortos que los requeridos en la forma tradicional.

- Que se logra compartir los riesgos del proyecto en forma equilibrada entre el concedente (el Estado) y el concesionario.

- Que la participación de empresas concesionarias modernas permite mejorar la calidad y cobertura de los servicios y la incorporación de nuevas tecnologías.

Sin embargo, revisando la experiencia de veinte ańos de concesiones en el Perú, se aprecia que, lamentablemente, estas ventajas no se están logrando en la mayor parte de los proyectos concesionados, conforme se discute a continuación. 


\section{Sobre la ventaja de trasladar el financiamiento del proyecto al concesionario}

La supuesta ventaja de ahorrar esfuerzos financieros al Estado concedente solo puede hacerse realidad siempre que el concesionario asuma plenamente el esfuerzo de inversión del proyecto. No obstante, esto no se viene dando en el caso de los megaproyectos, pues prácticamente en todos los casos se ha aplicado la figura del cofinanciamiento [2], en la que el concesionario no asume totalmente el financiamiento de la inversión, con lo que obliga al Estado a aportar parte importante de los costos. De esta manera, se desvirtúa la principal ventaja de las concesiones.

La aplicación del cofinanciamiento en los megaproyectos apareció implícita en el caso de la Línea 1 del Metro de Lima, cuando el Estado, a través del Ministerio de Transportes, asumió directamente la ejecución de la infraestructura pendiente en el tramo 2 ante la falta de interés de los posibles postores, quienes no veían atractivo asumir el financiamiento total del proyecto, ya que sería imposible recuperar una inversión tan cuantiosa con los ingresos futuros del servicio.

El cofinanciamiento en los contratos de concesión ha tenido, lamentablemente, sus expresiones más exageradas en el caso de la Línea 2 del Metro de Lima, donde el Estado -todos nosotros- asumió la mayor parte del financiamiento de uno de los trenes urbanos más caros del mundo, y también en el caso del Aeropuerto de Chinchero en el Cusco, donde se tenía un concesionario de dudosa capacidad financiera que pretendía que el Estado asuma la mayor parte de la inversión por adelantado y que, además, le pagara intereses por el atraso en la inversión pública.

Si el Estado va a asumir la mayor parte del financiamiento de un proyecto, ya no tiene sentido aplicar la modalidad de concesión. En ese sentido, parecería más lógico aplicar el anterior sistema de licitación con financiamiento, el cual existía en la antigua normativa de contrataciones de obras en el país. La licitación con financiamiento consiste en convocar a empresas interesadas en ejecutar las obras, a las que se les pide que, en sus ofertas, consideren la ejecución de los proyectos con financiamiento gestionado por ellas mismas, siendo el compromiso del Estado asumir el pago futuro de la deuda generada. Esta modalidad fue usada por la Municipalidad de Lima de manera exitosa en la ejecución de la Vía Expresa Javier Prado en el año 2000, y tuvo como favorable característica la posibilidad de precisar con mayor claridad la responsabilidad financiera del ejecutor del proyecto. Como es evidente, usando esta modalidad, el Estado logra la gran ventaja de trasladar el compromiso de financiamiento de la inversión a la empresa ejecutora sin necesidad de comprometerse con el ejecutor de las obras para encargarle la etapa operativa del proyecto, como en el caso en las concesiones.

En la licitación con financiamiento, el proceso se concentra en la etapa de ejecución sin incluir, en el mismo contrato, la administración futura del proyecto -lo que sí se tiene que hacer en el caso de las concesiones-. Esto permite que las bases para la selección del contratista ejecutor se simplifiquen, lo que facilita a los representantes de la entidad pública promotora, en resguardo del interés público, la selección del mejor ejecutor con adecuado financiamiento.

\section{Sobre la supuesta ventaja de ahorrar tiempo en el desarrollo de los proyectos}

Otra esgrimida ventaja de la modalidad de desarrollar proyectos mediante concesión es la del ahorro de tiempo para concretar y poner en operación las obras, pues al realizarse un único proceso de concurso de selección de concesionario - al que se encarga realizar los estudios y ejecutar el proyecto y la operación-supuestamente se requiere menos tiempo que en el procedimiento tradicional, en el que se deben llevar a cabo varios procesos: primero, de concurso, para seleccionar al consultor del estudio de preinversión; luego, para seleccionar al consultor para el estudio definitivo o expediente técnico; posteriormente, una etapa de licitación para seleccionar al ejecutor del proyecto; y, finalmente, otro concurso para seleccionar al operador. Sin embargo, es lamentable apreciar que esta esperada ventaja 
de las concesiones ha quedado desvirtuada totalmente en la práctica, pues, en todos los procesos de concurso de concesión, los cronogramas iniciales publicados en la convocatoria se han extendido por un exagerado número de ańos debido a la ineficiencia de los encargados de los procesos, los cuales siempre han convocado los concursos con bases muy defectuosas, las que luego se tienen que ir perfeccionando a lo largo de años. Así, se dilatan exageradamente los cronogramas de los procesos de selección de concesionarios, con lo que se pierde la esperada ventaja del ahorro de tiempo. En efecto, si miramos de manera panorámica la experiencia de todo este siglo en el desarrollo de proyectos de infraestructura mediante concesiones, se aprecian escasos resultados positivos y un importante número de experiencias negativas, entre las cuales se pueden remarcar las siguientes:

- La concesión de la Línea 1 del Metro de Lima, que presentó un proceso de selección de concesionario exageradamente dilatado, pues habiéndose asignado la responsabilidad del proceso a Proinversión en enero del 2007, el contrato de concesión recién se firmó en abril de 2011.

- El caso del Aeropuerto de Chinchero, que se decidió dar en concesión a inicios del año 2010, otorgándose la buena pro en abril del 2014, exagerado lapso de 4 ańos para seleccionar al concesionario, lo que evidencia la falta de capacidad profesional de los equipos de Proinversión, pues a lo largo de dichos ańos las bases de la convocatoria fueron modificadas decenas de veces.

- El caso de la línea 2 del Metro de Lima, cuya convocatoria se realizó en enero del 2013, y la buena pro se entregó en marzo del 2014, aparentemente con buen ahorro de tiempo, pero este ha quedado totalmente desvirtuado por el notable atraso en la ejecución de obra, la cual está actualmente trabada por problemas de diseño y de expropiaciones, todo ello consecuencia de insuficientes estudios de preinversión.

Precisamente, otra explicación a la excesiva dilación en los concursos de selección de concesionarios estaría en la carencia de estudios de preinversión completos, donde deben definirse muchos aspectos importantes del proyecto, en ausencia de los cuales se tienen que ir "madurando" las bases mediante un proceso de aproximaciones sucesivas, proceso que se aplica en los estudios de preinversión. No obstante, este procedimiento por tanteos no debe aplicarse en las fases de convocatoria y selección entre postores, a cargo de Pro inversión, ya que ofrece una lamentable imagen de pobreza profesional ante las empresas postoras y los inversionistas en general. Al respecto, se aprecia que el lamentable deseo de ahorrarse estudios parece una actitud perniciosa de los impulsores de los megaproyectos. En la experiencia de la mayor parte de las concesiones de obras públicas, se ha presentado la carencia de estudios de preinversión suficientemente profundos en discordancia con las importantes inversiones involucradas.

Todo proyecto de interés público debe contar con estudios de factibilidad completos, conforme recomienda la experiencia internacional. Sin embargo, esta importante recomendación ha sido mayormente soslayada, lo que ha dado lugar a procesos de concesión con estudios incompletos o deficientes y, en algunos casos, inexistentes. A nivel de factibilidad, la falta de adecuados estudios de preinversión provoca graves deficiencias en el desarrollo de los proyectos. Esto ocurre, por ejemplo, por no haberse detectado oportunamente la necesidad de realizar procesos de expropiación, como ocurrió con el caso del Aeropuerto de Chinchero, o con el caso extremo de la segunda pista del Aeropuerto Jorge Chávez, cuya construcción incluida como obligación del concesionario en el contrato del año 2000 estuvo prevista inicialmente para realizarse en el año 2006 y aún no comienza, pues de momento no se ha logrado el $100 \%$ de expropiación de los terrenos requeridos.

La carencia de estudios de factibilidad, donde se analice y decida las diversas alternativas de ingeniería conceptual de los proyectos, también significa un incremento innecesario en los costos de 
inversión de los proyectos -al tomarse importantes decisiones técnicas sin suficiente análisis-, como sucedió con la Línea 2 del Metro de Lima, propuesta en solución subterránea en todo su recorrido cuando, evidentemente, buena parte de ella podría desarrollarse en forma de viaducto elevado, de forma que su coste sea mucho más económico.

\section{El balance negativo de la experiencia de las concesiones en los megaproyectos}

De acuerdo con todo lo anterior, se aprecia que no se están logrando las esperadas ventajas de la modalidad de concesiones en cuanto a ahorrar esfuerzos financieros al Estado, y tampoco en cuanto a ahorrar tiempo en el desarrollo de los proyectos. En cambio, son muy notables las deficiencias en el desarrollo de los mismos, lo que implica mayores costos de inversión a ser asumidos por el Estado. Esta situación, a su vez, arroja serias dudas sobre la efectiva rentabilidad social de los proyectos.

Además, la experiencia de las concesiones de proyectos pone en evidencia las gruesas debilidades de la administración pública, cuya capacidad de gestión es superada por las exigencias que plantea el llevar cabo, de manera eficiente, complejos procesos de selección de concesionarios. Todo esto sucede en un ambiente de responsabilidades diseminadas, donde los proyectos están expuestos a los intereses políticos, lo que hace casi imposible garantizar el resguardo del interés público.

La débil capacidad de gestión de los representantes del interés público también se aprecia en los contratos concertados, donde no se logra la esperada ventaja de distribución equilibrada de los riesgos del proyecto entre el concedente (el Estado) y el concesionario, pues en los acuerdos se aprecia que el concesionario siempre cuenta con cláusulas de protección ante todo tipo de riesgos, mientras que no se explicitan claramente las previsiones para la protección del interés público. También, es notable la carencia de una gerencia centralizada por parte del Estado, que asuma la responsabilidad del seguimiento permanente de cada proyecto desde el proceso de selección del concesionario hasta la etapa de ejecución y operación, para evitar que se presenten situaciones que impidan el desarrollo normal de los proyectos, como es el caso de los procesos de expropiaciones.

\section{Recomendaciones para el mejor desarrollo de las concesiones de proyectos}

Parece conveniente revisar la política y la normatividad sobre el desarrollo de proyectos mediante concesiones, limitando su aplicación a proyectos de infraestructura debidamente estudiados y proponiendo algunos requisitos que serían imprescindibles para decidir la concesión de un megaproyecto:

- Que el proyecto propuesto para concesión cuente con estudios de factibilidad completos, con la ingeniería conceptual debidamente estudiada. A fin de neutralizar la presión política que se podría ejercer contra los consultores a cargo de los estudios y asegurar la rentabilidad social del proyecto, estos estudios deberían ser revisados por instituciones internacionales confiables como el BID o el Banco Mundial.

- Que en ningún caso se permita la aplicación del cofinanciamiento, ya que, si el Estado tiene que participar en las inversiones de un proyecto, y considerando que este no tiene suficiente rentabilidad financiera, será mejor aplicar la modalidad de licitación con financiamiento. De ninguna manera debe darse el cofinanciamiento en proyectos que nacen de iniciativas privadas.

- Que los procesos de concurso de concesión cuenten con bases adecuadamente preparadas, las que deberán contar con la revisión previa de especialistas de instituciones internacionales confiables como el BID o el Banco Mundial.

- Que se declare desierto el concurso para concesión cuando se llegue al final con postor único. 


\section{REFERENCIAS}

[1] A. Velásquez, "Experiencias de 15 Años de Concesiones en el Perú", Revista Paradigmas. Departamento de Ingeniería, 2011.

[2] Proinversión, Asociaciones Público-Privadas. [En línea]. Disponible en: http://www.proyectosapp.pe/modulos/JER/PlantillaStandard.aspx?are=0\&prf=2\&jer=5902 [Accedido: 05-jun-2017]. 